\title{
Reconfiguration and Load Shedding for Resilient and Reliable Multiple Microgrids
}

\author{
Kenneth Martin C. Atendido and Ramon Zamora \\ Electrical and Electronic Engineering Department \\ Auckland University of Technology \\ Auckland, New Zealand
}

\begin{abstract}
The microgrid concept sets the stage for an energy future consisting of networks of microgrids connected with one another. After a fault occurs, multiple interconnected microgrids engage the possibility of power transfer by reconfiguring the network topology to maximize the number of energized loads. This study proposes a two-stage restoration process by means of reconfiguration and load shedding. Reconfiguration is executed through a branch exchange of normally open tie lines while retaining radial topology. Load shedding is implemented with a particle swarm optimization post-reconfiguration to mitigate any load versus generation imbalance. The proposed algorithm was implemented using MATLAB and tested on a CERTS-based multiple microgrid modelled in DigSilent PowerFactory. All scenarios obtained satisfactory results where each indicated a maximum number of supplied loads and all that required load shedding presented reasonable amounts of load shed. This research contributes to multiple microgrid resiliency and reliability through reconfiguration and load shedding.
\end{abstract}

Keywords-CERTS, microgrid, reconfiguration, load shedding, PSO, DigSilent PowerFactory

\section{INTRODUCTION}

Sustained energy delivery despite the occurrence of sporadic events at a quality and quantity demanded by consumers characterizes a reliable and resilient power system. Natural disasters expose the weakness of power systems as consumers located far downstream of the electrical network are susceptible to massive shortages in power. This weakness, among others, has resulted in some of the worst power outages New Zealanders have experienced when natural disasters are imminent [1]. The scale of expansion of the electrical network, the imbalance between supply and demand, and susceptibility to outages from environmental disturbances may be mitigated through the penetration of distributed generation (DG) embedded across the distribution network. The idea of DG is to spread the generation of power throughout the network such that when certain areas experience interruptions, the rest of the network remain unaffected making the system more reliable and resilient.

Studies indicate however, that high penetration of DG in power systems may produce as many problems as it solves. To mitigate these issues and realize the emerging potential of DG, researchers have concentrated on taking a systematic approach which views generation and associated loads as a subsystem or a "microgrid" [2]. Microgrids research is trending as it presents a viable means of congestion relief, postponement of new generation, response to load changes and local voltage support.

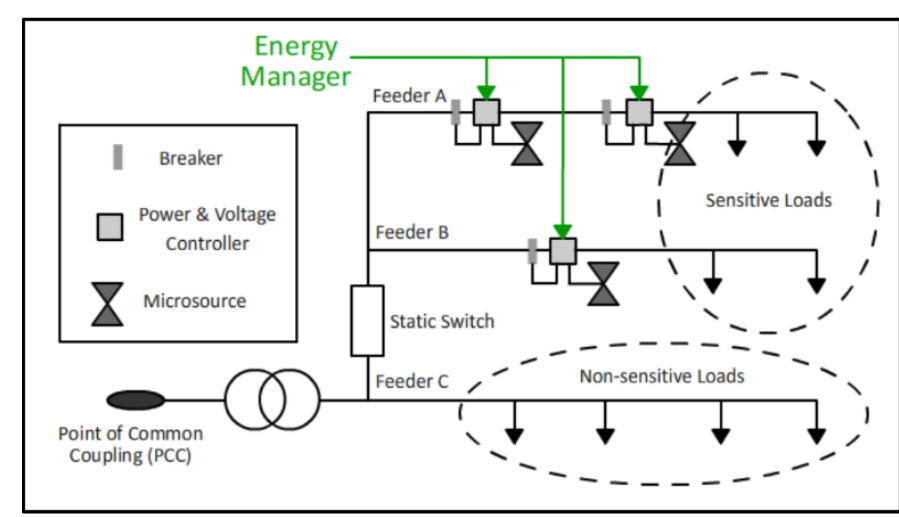

Fig. 1. CERTS microgrid architecture

Microgrid research readily available today are typically in the fields of load management, protection schemes, control strategies, optimized capacitor/DG location, optimal operation during islanded mode, power scheduling, communication systems etc. [3-6].

The most commonly known microgrid architecture is the Consortium for Electric Reliability Technology Solutions (CERTS) architecture shown in Fig. 1 [2]. The CERTS defines a microgrid as a radial distribution system with several feeders and a collection of microsources and loads [7]. It is connected to the main grid via the point of common coupling (PCC) and can operate in grid-connected or islanded mode. The interest in microgrids is trending due to the increasing consumption of traditional energy (centralized generation) and rising concern for the environment. Microgrid research interest has grown because of their significant advantages such as providing environmentally friendly, secure, reliable, efficient, and sustainable electricity to the distribution network [2].

\section{PROBLEM FORMULATION}

Intermittent events such as natural disasters make microgrids susceptible to outages and disturbances. Microgrid reconfiguration is a necessary plan of action which allows for resiliency and reliability of the network. Despite the numerous research material on microgrids, limited research has focused on reconfiguration strategies in conjunction with load shedding especially for a multiple microgrid system. The concept of multiple microgrid reconfiguration aims to minimize load 
interruption enabling self-healing for greater reliability and resiliency of the distribution system.

Reconfiguration is a necessary aspect of operation as it increases the survivability of system functionality when unexpected events occur. This is achieved through normally open tie lines between feeders and, in this study, microgrids. These lines may be closed when other lines are out of service to restore connectivity allowing for a more resilient system. By successfully implementing these reconfiguration strategies, this study hopes to lessen the impact of disturbances and increase the recoverability of the microgrids during unfortunate events.

After reconfiguration is implemented, some loads could potentially be left undersupplied. Generators and/or lines could be overloaded by too much demand and bus voltages could drop below operational limits as well. Load shedding relieves the system from operating beyond or below its nominal range to prevent any potential large-scale disturbance. This strategy reallocates the scarce energy available from lower priority loads to loads with either high priority or on the brink of causing a fault. Reconfiguring the microgrid to adapt to these instances will increase its resiliency and reliability.

Load prioritization will be considered by categorizing customer loads on a priority level system. There can be numerous rules or constraints when categorizing a load's priority. A simple example used in [8] categorizes loads under priority and non-priority. Priority loads were defined as loads that when left unsupplied could endanger human life or safety such as hospital emergency rooms and intensive care units. Nonpriority loads are the opposite in that when left unsupplied would not endanger human life or safety such as corporate office spaces, retail stores or residential loads. Further indices could be provided for more specific levels of priority which could further define the degree of priority or non-priority load/s.

\section{Multiple MiCROGRID ReCONFIGURATION}

Reconfiguration mitigates any disconnection by finding the best configuration that would interconnect all nodes in the event of an outage while satisfying operational constraints. The microgrid system adopted in this study is modelled in DigSilent PowerFactory (DSPF) and based on modifications of CERTS microgrid systems available in [8-11] as presented in Fig. 2.

A breadth-first search method was implemented to map out the nodes and determine which are disconnected from the main network. Branch exchange follows for all normally open tie lines to determine which tie line could possibly reconnect islanded node/s. It should be noted that the search space for the

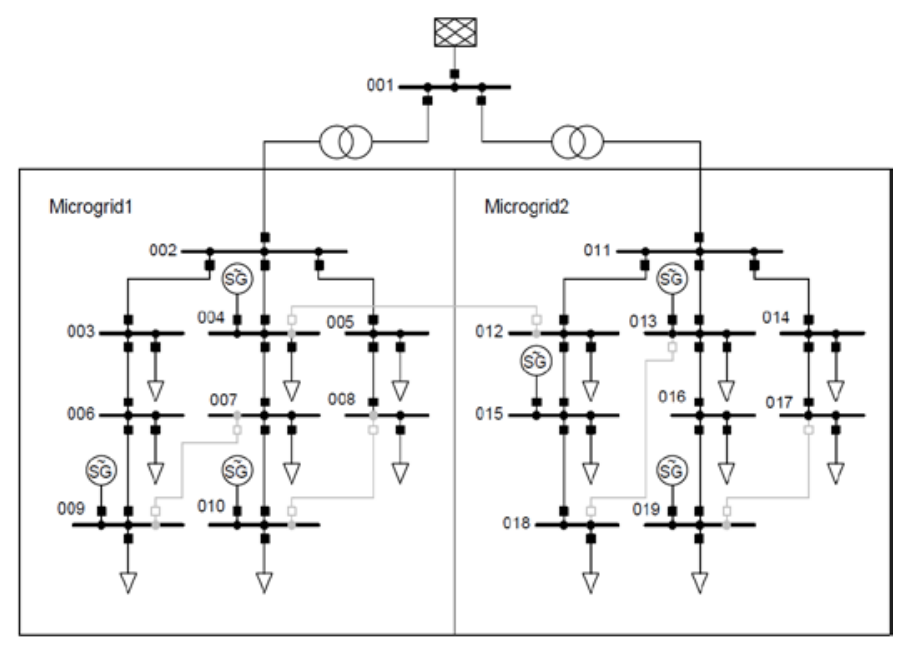

Fig. 2. CERTS-based multiple microgrid model reconfiguration is simply the number given by closing tie lines. This is given by (1).

$$
C=2^{T}
$$

where, $C$ refers to the number of possible configurations and $T$ pertains to the number of open tie lines. At this point, load priority is not considered because the main goal of this reconfiguration is to ensure all islanded nodes are reconnected back to the network.

It is possible to obtain new configurations that meet topological requirements but when implemented may not meet operational constraints. Some methods would typically disregard these potential solutions due to the lack of another stage in the restoration process such as $[12,13]$. Since this study combines reconfiguration with load shedding, non-convergent solutions that have recombined all islanded nodes would simply pertain to overloading of either lines or generating nodes and/or a violation of bus voltage limits. This would be addressed in Section IV pertaining to load shedding.

The objective of this reconfiguration is to maximize the number of reconnected load nodes as given in (2).

$$
\max \sum_{i=1}^{l}\left(P_{L, i} y_{i}\right)
$$

where $l$ is the number of load buses, $P_{L, i}$ is the load power consumed at bus $i$, and $y_{i}$ is the status of load bus $i$ ( 1 or 0 ).

The reconfiguration begins with an initialization of a list of possible line switches for all tie lines. The power flow is taken for each possible configuration and the power mismatch and amount of power loss is used to determine the fitness of the new topology. Loop detection is implemented to ensure each configuration is radial. Priority configurations are characterized as the topologies that are both radial and achieve a convergent power flow because this suggests that the operational constraints are met, and no further action is potentially required. Configurations whose power flow is non-convergent are set aside and used as input to the load shedding algorithm, described in the next section, if no convergent solution is obtained.

\section{APPLICATION OF PSO IN LOAD SHEDDING}

PSO was introduced by Kennedy and Eberhart in 1995 in their paper entitled, Particle Swarm Optimization [14]. The PSO is a swarm intelligence paradigm that mimics the behavior of flocking birds or schooling fish to guide particles to search for globally optimal solutions. The individuals or particles in a particle swarm "evolve" by cooperation and competition among other individuals in the population and other generations.

\section{A. General PSO}

In PSO, each individual is treated like a particle in a search space. The swarm initially consists of randomized variables, bounded by a certain upper and lower limit. The fitness of each particle in the swarm or population is then calculated based on the specified objective function and corresponding penalty function for any violation of indicated constraints. These particles each have a dynamically adjusted velocity that is updated based on its own experience and the experience of its neighbors. The velocity of the particle indicates the learning process behind PSO and is given by (3). The position is recalculated based on the new velocity and is given by (4). A dynamic inertia weight was used in this study as shown in (5), because $[14,15]$ indicate that faster and more consistent results 
may be obtained for a minimum and maximum of 0.9 and 1.2, respectively.

$$
\begin{array}{r}
V_{i, j}^{k+1}=w \times V_{i, j}^{k}+\alpha r(t) \times\left(\text { Pbest }_{i, j}^{k}-X_{i, j}^{k}\right) \\
+\beta r(t) \times\left(\text { Gbest }_{j}^{k}-X_{i, j}^{k}\right), \\
X_{i, j}^{k}=X_{i, j}^{k}+V_{i, j}^{k+1}, \\
w=w_{\text {max }}-\left(w_{\text {max }}-w_{\text {min }}\right) \times \frac{k}{\text { maxiter }},
\end{array}
$$

where $V_{i, j}^{k}$ is the particle velocity at iteration $k, X_{i, j}^{k}$ is the position of the $i^{\text {th }}$ component of $j^{\text {th }}$ individual in population at iteration $k, \alpha, \beta$ are acceleration coefficients, $r$ is a random uniform distribution value between 0 and 1 , and $w$ is the inertia weight.

From [8, 14, 16, 17], the suggested value for each acceleration coefficient is on average the integer value 2 . These are normally unchanged even with varying applications.

\section{B. Fitness Function}

PSO was implemented in this study to conduct load shedding due to its ease of use and quick computation. This study would like to investigate the operation of an adaptive method of load shedding across a distribution system to determine whether such an approach is comparable to traditional methods of load shedding such as the use of automatic relays.

The variables used in this PSO are the operating capacities of each load bus in the multiple microgrid system, where a decrease in load capacity corresponds to a switching action on a load node's available breakers. This means that the trajectory of each particle pertains to the amount of load shed a particular node could experience. For a search space consisting of multiple discrete load shedding steps for all available load buses, this PSO has approximately $2.38 \times 10^{8}$ possible combinations of load shed switch statuses. To determine the fitness of each particle's set of load capacity values, this algorithm aims to minimize the objective function in (6).

$$
\min \sum_{i=1}^{l}\left(P_{\text {Lnom }, i}-P_{\text {Loper }, i}\right) \rho_{i}
$$

where $l$ is the number of load buses, $P_{\text {Lnom, } i}$ is the peak load power demand in $\mathrm{kVA}$ at bus $i, P_{\text {Loper }, i}$ is the operating load power in $\mathrm{kVA}$ at bus $i$, and $\rho_{i}$ is the load bus priority index.

Multiple levels of load prioritization were introduced in this study to demonstrate the capability of an adaptive method to allocate load shedding accordingly. These priority indices represent the magnitude of importance of a load and were arbitrarily segregated into five (5) levels namely: important (75), somewhat important (50), neutral (15), somewhat unimportant (10), and unimportant (1). The load parameters for the CERTS-based model used in this study are detailed in Table I.

The process of this PSO-based load shedding is as follows:

1. Set population size and maximum iteration number.

2. Randomly generate load values and assign to $X_{i, j}^{k}$.

3. Set population size and maximum iteration number.

4. Randomly generate load values and assign to $X_{i, j}^{k}$
TABLE I. LOAD PARAMETERS

\begin{tabular}{|c|c|c|c|c|}
\hline Load & Priority & $\begin{array}{c}\text { Peak } \\
\text { Demand } \\
\text { (kVA) }\end{array}$ & $\begin{array}{c}\text { \# of 5kVA } \\
\text { Steps }\end{array}$ & $\begin{array}{c}\text { \# of 10kVA } \\
\text { Steps }\end{array}$ \\
\hline 3 & 50 & 50 & 3 & - \\
\hline 4 & 75 & 105 & 2 & - \\
\hline 5 & 10 & 70 & - & 4 \\
\hline 6 & 1 & 35 & - & 3 \\
\hline 7 & 15 & 70 & 1 & 3 \\
\hline 8 & 1 & 35 & - & 3 \\
\hline 9 & 50 & 50 & 3 & - \\
\hline 10 & 50 & 50 & 3 & - \\
\hline 12 & 1 & 35 & - & 3 \\
\hline 13 & 15 & 35 & 3 & 3 \\
\hline 14 & 1 & 35 & - & - \\
\hline 15 & 15 & 35 & 3 & 3 \\
\hline 16 & 1 & 35 & - & 3 \\
\hline 17 & 1 & 35 & - & - \\
\hline 18 & 1 & 35 & - & \\
\hline 19 & 75 & 105 & 2 & \\
\hline
\end{tabular}

5. Calculate initial fitness based on objective function and penalties from violated constraints namely:

$$
\begin{array}{rlrl}
P_{G \min , i} & \leq P_{G, i} \leq P_{G \max , i} & i=1, \ldots, N_{G e n} \\
Q_{G \min , i} \leq Q_{G, i} \leq Q_{G \max , i} & i=1, \ldots, N_{G e n} \\
V_{L m i n, i} \leq V_{L, i} \leq V_{\text {Lmax }, i} & i=1, \ldots, N_{\text {Load }} \\
S L_{\text {min }, i} \leq S L_{i} \leq S L_{\text {max }, i} & i=1, \ldots, N_{\text {Line }}
\end{array}
$$

where $P_{G, i}$ is active power capacity of DG at bus $i$ in $k W, Q_{G, i}$ is reactive power capacity of DG at bus $i$ in $k W, V_{L, i}$ is voltage at bus $i$ in per unit voltage, and $S L_{i}$ is line limit at line $i$ in $k W$. Assign as initial personal best and global best.

6. Update velocity and position of particles using (3)-(5).

7. Calculate fitness of new particles. If fitness of new particle is better than the previous, then update the personal best. If the best particle in the current population is better than the previous population, then update the global best.

8. If maximum iteration number is exceeded, optimal solution is the global best.

In this study, the protection system isolates any fault by tripping breakers using typical fault detecting functions. MATLAB is then used to implement a reconfiguration and load shedding algorithm. The reconfiguration begins with a breadthfirst search method that maps out the grid network and determines any islanding node/s. If any islanding node/s are identified, a branch exchange of all open tie lines follows to identify possible paths that may provide added power to neighboring microgrids or unsupplied load nodes. A loop detection technique was adopted from the Iterative Loop Counting Algorithm (ILCA) presented in [18] to determine whether the possible configuration remains radial or not. Operating in a loop configuration when no power is circulated back is simply unnecessary due to the assumed greater load demand than the available generation from DG hence, a radial configuration is maintained. The power flow of the chosen network configuration is then taken to determine any load versus generation imbalance. An intelligent algorithm in the form of PSO is used to solve for an optimal amount of load shed to address the power imbalance. The final results are imported into DSPF for verification. The process is shown in Fig. 3. 


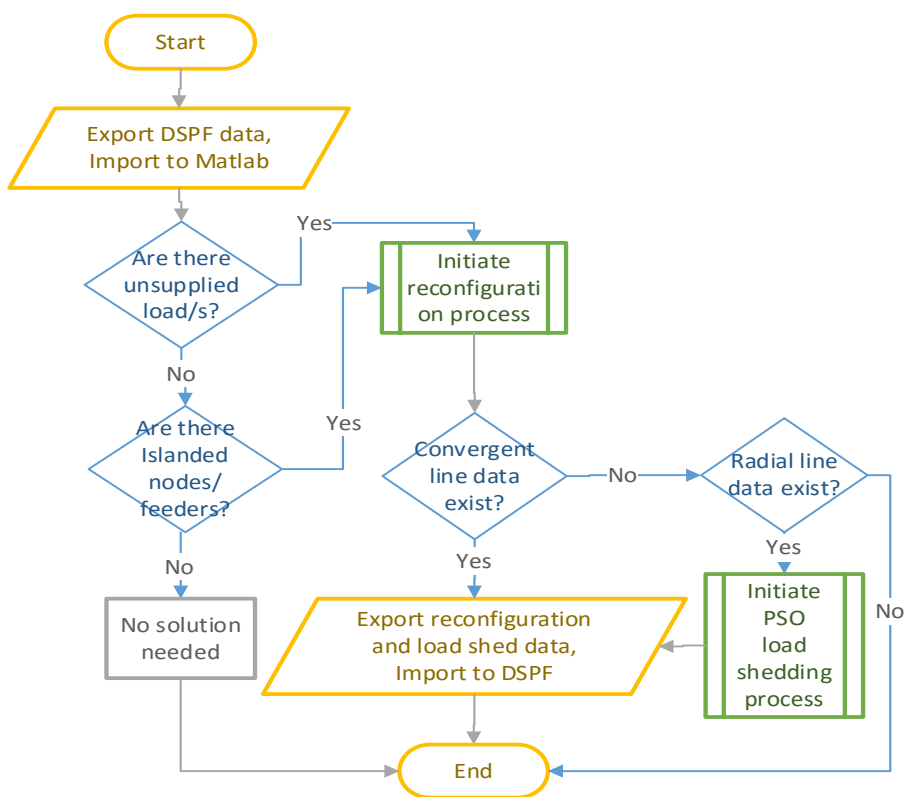

Fig. 3. Flowchart of reconfiguration and load shedding process

\section{RESUlTS AND DISCUSSION}

A population size of 20 and maximum iteration number of 30 was implemented in the PSO-based load shedding algorithm. Generation and line parameters are outlined in Tables II and III. Each line consists of a breaker that is normally closed except for the tie lines which are normally open as seen in Fig. 2. The line numbers indicated in Table III will be used as the reference name connecting the buses hereafter i.e. bus 1 to 2 will be referred to as Line 1 , bus 1 to 11 as Line 2 and so on.

Four case scenarios were simulated in MATLAB and DSPF. One or two network elements were placed out of service except for case 4 which was included as a means of presenting the capabilities of the algorithm. The results are shown in Table IV. The final line switch configuration column indicates the resulting network topology post-reconfiguration with the following column indicating how many switching actions took place in the reconfiguration. The load shedding column indicates the bus number of loads that required incremental shedding, with the final column presenting how much total load was consequently served. Each case scenario demonstrates the capability of the developed algorithm to identify the need for reconfiguration and/or load shedding and whether the processes produce a final solution that meets operational constraints.
Despite indicating load priority in the objective function, the PSO did not appear to hesitate shedding higher priority loads. What happened instead was that loads with high priority only shed a small amount. This was quite understandable because whether a load is of high priority, if it needed to be shed for the system to meet constraints, then it is a necessary action. The most load shed was experienced by case 3 due to the lack of external grid support caused by the simulated fault. From Table I, the total load demand at each microgrid is far greater than the total capacity of DGs present in each microgrid, therefore the deficit must be shed accordingly.

TABLE II. DISTRIBUTED GENERATION CAPACITIES

\begin{tabular}{|c|c|c|}
\hline Microgrid & DG Bus no. & Capacity (kVA) \\
\hline \multirow{3}{*}{1} & 4 & 120 \\
\cline { 2 - 3 } & 9 & 100 \\
\cline { 2 - 3 } & 10 & 120 \\
\hline \multirow{3}{*}{2} & 13 & 100 \\
\cline { 2 - 3 } & 15 & 100 \\
\cline { 2 - 3 } & 19 & 100 \\
\hline
\end{tabular}

TABLE III. LINE PARAMETERS

\begin{tabular}{|c|c|c|c|c|c|}
\hline Line No. & $\begin{array}{c}\text { From } \\
\text { Bus No. }\end{array}$ & $\begin{array}{c}\text { To } \\
\text { Bus No. }\end{array}$ & $\mathbf{R}(\Omega)$ & $\mathrm{X}(\mathbf{\Omega})$ & Switch Status* \\
\hline 1 & 1 & 2 & 0.000 & 0.200 & 1 \\
\hline 2 & 1 & 11 & 0.000 & 0.200 & 1 \\
\hline 3 & 2 & 3 & 0.045 & 0.186 & 1 \\
\hline 4 & 2 & 4 & 0.045 & 0.186 & 1 \\
\hline 5 & 2 & 5 & 0.047 & 0.100 & 1 \\
\hline 6 & 3 & 6 & 0.047 & 0.100 & 1 \\
\hline 7 & 4 & 7 & 0.047 & 0.100 & 1 \\
\hline 8 & 4 & 12 & 0.090 & 0.037 & 0 \\
\hline 9 & 5 & 8 & 0.047 & 0.100 & 1 \\
\hline 10 & 6 & 9 & 0.047 & 0.100 & 1 \\
\hline 11 & 7 & 9 & 0.090 & 0.037 & 0 \\
\hline 12 & 7 & 10 & 0.047 & 0.100 & 1 \\
\hline 13 & 8 & 10 & 0.090 & 0.037 & 0 \\
\hline 14 & 11 & 12 & 0.045 & 0.186 & 1 \\
\hline 15 & 11 & 13 & 0.045 & 0.186 & 1 \\
\hline 16 & 11 & 14 & 0.047 & 0.100 & 1 \\
\hline 17 & 12 & 15 & 0.047 & 0.100 & 1 \\
\hline 18 & 13 & 16 & 0.047 & 0.100 & 1 \\
\hline 19 & 13 & 18 & 0.090 & 0.037 & 0 \\
\hline 20 & 14 & 17 & 0.047 & 0.100 & 1 \\
\hline 21 & 15 & 18 & 0.047 & 0.100 & 1 \\
\hline 22 & 16 & 19 & 0.047 & 0.100 & 1 \\
\hline 23 & 17 & 19 & 0.090 & 0.037 & 0 \\
\hline
\end{tabular}

*Status of 1 indicates closed whereas 0 indicates open

TABLE IV. POST-RECONFIGURATION AND LOAD SHEDDING RESULTS

\begin{tabular}{|c|c|c|c|c|c|}
\hline Test Case & $\begin{array}{c}\text { Faulted } \\
\text { Element } / \mathrm{s}\end{array}$ & Final Line Switch Configuration & No. of Switching & Load Shedding & kW Served \\
\hline Base Case & - & $\begin{array}{c}\text { Closed: } 1,2,3,4,5,6,7,9,10,12, \\
\text { 14, 15, 16, 17, 18, 20, 21, } 22 \\
\text { Open: } 8,11,13,19,23\end{array}$ & - & - & 815 \\
\hline Case 1 & Line 1 & $\begin{array}{c}\text { Closed: } 2,3,4,5,6,7,8,9,10,12, \\
14,15,16,17,18,20,21,22 \\
\text { Open: } 11,13,19,23\end{array}$ & 1 & $5,6,8,9,12,17$ & 680 \\
\hline Case 2 & $\begin{array}{c}\text { Line 2, Line } \\
16\end{array}$ & $\begin{array}{c}\text { Closed: } 1,3,4,5,6,7,8,9,10,12, \\
14,15,17,18,20,21,22,23 \\
\text { Open: } 11,13,19\end{array}$ & 2 & $14,15,16,17,18$ & 720 \\
\hline Case 3 & $\begin{array}{l}\text { Main grid } \\
\text { PCC }\end{array}$ & $\begin{array}{c}\text { Closed: } 1,2,3,4,5,6,7,9,10,12, \\
\text { 14, 15, 16, 17, 18, 20, 21, } 22 \\
\text { Open: } 8,11,13,19,23\end{array}$ & - & $\begin{array}{c}3,4,5,6,7,8,9,10,12,1 \\
3,14,15,16\end{array}$ & 630 \\
\hline Case 4 & $\begin{array}{l}\text { Gen bus } 15 \text {, } \\
\text { Line } 14 \text {, Line } \\
21\end{array}$ & $\begin{array}{c}\text { Closed: } 1,2,3,4,5,6,7,8,9,10,12 \\
\text { 15, } 16,17,18,19,20,22 \\
\text { Open: } 11,13,23\end{array}$ & 2 & - & 815 \\
\hline
\end{tabular}


The proposed reconfiguration and load shedding algorithms were tested on a CERTS-based multiple microgrid model in MATLAB and DSPF. The reconfiguration detects islanded node/s through a breadth-first search traversing through the network topology in search of unconnected nodes. The detected islanding is then addressed by a branch exchange of normally open tie lines. Any ensuing load versus generation imbalance is solved by the PSO-based load shedding algorithm. This will run across the best reconfiguration obtained and will calculate for the most optimal solution minimizing the amount of load shed. The results of the simulation indicated maximized reconnection of load nodes and modest load shedding.

\section{CONCLUSION}

Research on multiple microgrid operation for grid reliability and resiliency was conducted in this work through the implementation and development of a reconfiguration and load shedding algorithm. A branch exchange-based reconfiguration was proposed and implemented to minimize unsupplied loads while maintaining radial configuration. The reconfiguration search space consisted of all combinations of these tie lines being closed and opened. Loop detection and power flow techniques were employed to ensure the obtained reconfigurations satisfy constraints and remain radial. A PSObased load shedding was proposed as a subsequent process to address any load and generation imbalance consequent to faults and/or reconfiguration. Compared to automatic load shedding, the adaptiveness of the PSO in this study allows for load to be shared rather than removed completely. Despite requiring load shedding to bring the system to a secure state of operation, the PSO aimed to minimize the amount of load shedding based on five predetermined load priority indices. All processes were constrained to bus voltage, line, and generation limits. These were simulated on a modified CERTS-based multiple microgrid network consisting of 19 buses, 23 lines (5 tie lines), 16 loads and 6 DG units. All results confirm the proposed algorithm's contribution to grid resiliency and reliability by enabling the maximization of supplied loads and minimizing load shed.

\section{ACKNOWLEDGMENT}

The authors would like to thank AUT for all the resources provided for this research.

\section{REFERENCES}

[1] M. Field and L. Walters. (2014, 27-Aug 2017). Auckland's history of power cuts. Available: http://www.stuff.co.nz/national/10583844/Aucklandshistory-of-power-cuts

[2] R. Zamora and A. K. Srivastava, "Controls for microgrids with storage: Review, challenges, and research needs," Renewable and Sustainable Energy Reviews, vol. 14, pp. 2009-2018, 2010/09/01/ 2010.

[3] Z. Xiaobo, G. Jun, and Z. Baohui, "A master slave peer to peer integration microgrid control strategy based on communication," in 2016 IEEE PES Asia-Pacific Power and Energy Engineering Conference (APPEEC), 2016, pp. 1106-1110.

[4] X. Zhu and K. C. Leung, "An adaptive distributed power scheduling algorithm for renewable Microgrid cooperation," in 2017 IEEE International Conference on Communications (ICC), 2017, pp. 1-6.

[5] D. Kumar, F. Zare, and A. Ghosh, "DC Microgrid Technology: System Architectures, AC Grid Interfaces, Grounding Schemes, Power Quality, Communication
Networks, Applications, and Standardizations Aspects," IEEE Access, vol. 5, pp. 12230-12256, 2017.

[6] D. Yuanxiao, B. Chen, Q. Li, and J. Liu, "Control strategy of low voltage microgrid based on equivalent reference current control," in 2014 China International Conference on Electricity Distribution (CICED), 2014, pp. 70-76.

[7] I. Patrao, E. Figueres, G. Garcerá, and R. GonzálezMedina, "Microgrid architectures for low voltage distributed generation," Renewable and Sustainable Energy Reviews, vol. 43, pp. 415-424, 2015/03/01/ 2015.

[8] F. A. Martina, "Graph Theory and Particle Swarm Based Reconfiguration of Multiple Microgrids for Grid Resiliency," Master of Science in Electrical Engineering Masters, School of Electrical Engineering \& Computer Sciences, Washington State University, Washington, USA, 2014.

[9] R. Lasseter, A. Akhil, C. Marnay, J. Stevens, J. Dagle, R. Guttromson, et al., "The CERTS microgrid concept, white paper on integration of distributed energy resources," California Energy Commission, Office of Power Technologies-US Department of Energy, LBNL50829, http://certs. Ibl. gov, 2002.

[10] F. Shariatzadeh, R. Zamora, and A. K. Srivastava, "Real time implementation of microgrid reconfiguration," in 2011 North American Power Symposium, 2011, pp. 1-6.

[11] F. Shariatzadeh, N. Kumar, and A. K. Srivastava, "Intelligent control algorithms for optimal reconfiguration of microgrid distribution system," in Industry Applications Society Annual Meeting, 2015 IEEE, 2015, pp. 1-8.

[12] M. E. Baran and F. F. Wu, "Network reconfiguration in distribution systems for loss reduction and load balancing," IEEE Transactions on Power Delivery, vol. 4, pp. 1401-1407, 1989.

[13] L. S. M. Guedes, A. C. Lisboa, D. A. G. Vieira, and R. R. Saldanha, "A Multiobjective Heuristic for Reconfiguration of the Electrical Radial Network," IEEE Transactions on Power Delivery, vol. 28, pp. 311319, 2013.

[14] J. Kennedy, "Particle swarm optimization," in Encyclopedia of machine learning, ed: Springer, 2011, pp. 760-766.

[15] Y. Shi and R. Eberhart, "A modified particle swarm optimizer," in Evolutionary Computation Proceedings, 1998. IEEE World Congress on Computational Intelligence., The 1998 IEEE International Conference on, 1998, pp. 69-73.

[16] Z. H. Zhan, J. Zhang, Y. Li, and H. S. H. Chung, "Adaptive Particle Swarm Optimization," IEEE Transactions on Systems, Man, and Cybernetics, Part B (Cybernetics), vol. 39, pp. 1362-1381, 2009.

[17] G.-y. Wang and D.-x. Han, "Particle Swarm Optimization Based on Self-adaptive Acceleration Factors," presented at the WGEC '09. 3rd International Conference on Genetic and Evolutionary Computing, 2009. , Guilin, China, 2009.

[18] J. Kirk. (2012, 26 July 2017). Count Loops in a Graph. Available:

http://au.mathworks.com/matlabcentral/fileexchange/1 0722-count-loops-in-a-graph 